\title{
The impact of pathologic staging of the hilar/mediastinal nodes on outcomes in patients with early-stage NSCLC receiving stereotactic body radiotherapy
}

\author{
Brandon T. Mullins ${ }^{1}$, Dominic T. Moore ${ }^{1,2}$, M. Patricia Rivera ${ }^{2,3}$, Lawrence B. Marks ${ }^{1,2}$, Jason Akulian ${ }^{2,3}$, \\ Kevin A. Pearlstein ${ }^{1,2}$, Kyle Wang ${ }^{1,2}$, Allen C. Burks ${ }^{2,3}$, Ashley A. Weiner ${ }^{1,2}$ \\ ${ }^{1}$ Department of Radiation Oncology, University of North Carolina Hospitals, Chapel Hill, NC, USA; ${ }^{2}$ Lineberger Comprehensive Cancer Center, \\ University of North Carolina at Chapel Hill, Chapel Hill, NC, USA; ${ }^{3}$ Department of Medicine, Division of Pulmonary and Critical Care Medicine, \\ University of North Carolina at Chapel Hill, Chapel Hill, NC, USA \\ Contributions: (I) Conception and design: BT Mullins, MP Rivera, J Akulian, AA Weiner; (II) Administrative support: LB Marks, AA Weiner; (III) \\ Provision of study materials or patients: LB Marks, MP Rivera, J Akulian, KA Pearlstein, K Wang, AC Burks, AA Weiner; (IV) Collection and \\ assembly of data: BT Mullins, KA Pearlstein, AA Weiner; (V) Data analysis and interpretation: DT Moore, BT Mullins, AA Weiner; (VI) Manuscript \\ writing: All authors; (VII) Final approval of manuscript: All authors. \\ Correspondence to: Ashley A. Weiner, MD, PhD. Department of Radiation Oncology, University of North Carolina Hospitals, 101 Manning Drive, \\ Chapel Hill, NC 27514, USA. Email: ashley_weiner@med.unc.edu.
}

Background: The importance of invasive mediastinal nodal staging in early-stage non-small cell lung cancer (NSCLC) in the PET/CT era is dependent on tumor factors that increase risk of nodal metastasis. At our institution, patients undergo biopsy via either CT-guidance (without nodal staging) or navigational bronchoscopy with endobronchial ultrasound transbronchial needle aspiration for nodal staging. This study aims to compare outcomes after stereotactic body radiotherapy (SBRT) stratified by receipt of invasive mediastinal nodal staging.

Methods: In this retrospective study, records of all consecutive patients undergoing SBRT for earlystage NSCLC between 2010 and 2017 were analyzed. The association between time-to event outcomes (recurrence and survival) were evaluated with covariates of interest including tumor size, location, histology, smoking history, prior lung cancer history, radiation dose and receipt of nodal staging. Both univariable and multivariable analyses were used to examine these comparisons.

Results: Overall, 158 patients were treated with SBRT. One hundred forty-nine out of one hundred fifty-eight patients (94\%) underwent PET/CT staging, and all patients underwent tumor-directed biopsy. Seventy-nine patients underwent navigational bronchoscopy with nodal staging and 79 patients underwent CT-guided biopsy without nodal staging. Receipt of nodal staging was not associated with tumor size $(\mathrm{P}=0.35)$, yet was associated with central tumor location $(\mathrm{P}<0.001)$. There was no statistically significant association between receipt of nodal staging and time-to-event recurrence or survival outcomes; for example 3-year overall survival (OS) was $65 \%$ vs. $67 \%(\mathrm{P}=0.65)$ and 3 -year freedom from nodal failure was $84 \%$ vs. $69 \%$ $(\mathrm{P}=0.1)$ for those with and without nodal staging, respectively.

Conclusions: Similar recurrence and survival outcomes were observed after SBRT regardless of receipt of invasive mediastinal nodal staging. Further prospective evaluation can help identify which patients might derive greatest benefit from invasive staging of the mediastinum in the PET/CT era.

Keywords: Non-small cell lung cancer (NSCLC); stereotactic body radiotherapy (SBRT); nodal staging; lung cancer; radiation therapy

Submitted Sep 03, 2020. Accepted for publication Dec 10, 2020.

doi: $10.21037 /$ jtd-20-2808

View this article at: http://dx.doi.org/10.21037/jtd-20-2808 


\section{Introduction}

In non-small cell lung cancer (NSCLC), accurate staging guides appropriate treatment selection and optimal patient outcomes. Medically operable patients with node-negative (or N1-positive disease) may proceed to surgical resection, while patients with mediastinal lymph node involvement often require multimodality therapy. For patients with small primary tumors and clinically presumed early-stage disease, if a surgical approach is selected, lobectomy is usually preceded by histologic nodal assessment (e.g., mediastinal lymph node biopsy or dissection). However, if these same patients receive non-operative therapy (due to medical comorbidities and/or patient choice) with stereotactic body radiation therapy (SBRT) (1-4), the lymph nodes are not necessarily assessed pathologically (as they would be during surgical resection).

Pathologic assessment of hilar and mediastinal nodal stations (invasive mediastinal nodal staging) is performed via either endobronchial ultrasound with transbronchial needle aspiration (EBUS-TBNA) or mediastinoscopy, with an accuracy of approximately $89 \%$ and $100 \%$, respectively (5-7). In the PET/CT era, the necessity of formal pathologic nodal assessment in clinical early-stage disease has been less clear given the accuracy of PET/CT (with negative predictive values $>80-90 \%$ ), leading many to avoid pathologic assessment of clinically/radiographically negative nodes $(5,8-13)$. However, PET/CT cannot adequately detect micro-metastatic disease $(9,10)$; and thus, one might expect better outcomes in patients who have their nodes histologically assessed compared to those who do not.

At our institution, some medically inoperable patients treated with SBRT undergo pathologic assessment of their nodes, while others have nodal assessment with only PET/ CT. We herein compare time-to-event outcomes in our SBRT patients based on receipt of invasive mediastinal nodal staging. We present the following article in accordance with the STROBE reporting checklist (available at http://dx.doi.org/10.21037/jtd-20-2808).

\section{Methods}

\section{Patient population}

The records of 158 consecutive adult patients (with 179 tumors) undergoing SBRT using Cyberknife (Accuracy) for early-stage NSCLC at the University of North Carolina Hospitals from 2010-2017 were retrospectively analyzed as part of an Institutional Review Board-approved study
(IRB\# 14-2566). Select patient demographics including: age at diagnosis, sex, performance status, tumor characteristics and treatment details were obtained through medical record abstraction.

\section{Treatment parameters}

Prior to SBRT simulation, gold fiducial markers for tumor tracking were implanted inside or near the tumor using either CT-guidance or navigational bronchoscopy. Simulation was performed with patients in supine position, with arms at sides, using a custom-made immobilization device. To aid lesion tracking during respiration, patients wore the Cyberknife "synchrony" tracking vest. Breath hold CT scan (1 mm thin images) was used for contouring the gross tumor volume (GTV) with a 4D CT scan obtained to aid tumor volume delineation with respiratory motion changes. GTV was contoured using lung windows, and was expanded typically by $5 \mathrm{~mm}$ radially and $8 \mathrm{~mm}$ superior/ inferior to define the planning target volume (PTV). Treatment plans were generated using Cyberknife nonisocentric, inverse-planning with radiation dose delivered to an isodose line with at least $\approx 95 \%$ PTV coverage. For posttreatment surveillance, CTs were typically obtained every 3-4 months for the first two years after SBRT, and every 6-8 months for years three through five, and then once annually after that.

\section{Data assessment}

Invasive mediastinal nodal staging receipt was defined as those undergoing navigational bronchoscopy with EBUSTBNA of visualized lymph nodes. Tumor size, location, appearance (solid $v s$. sub-solid), and the presence or absence of a suspicious lymph node on PET/CT were determined by direct review of each patient's chest imaging and validated with the radiology report. Typically, lymph nodes were labelled as "suspicious" when FDG uptake was greater than blood pool, but less than hepatic parenchyma. Tumor staging was based on the AJCC $8^{\text {th }}$ edition tumornode-metastasis (TNM) staging system (14). Central tumor location was defined as all tumors within $2 \mathrm{~cm}$ of the proximal bronchial tree or within $2 \mathrm{~cm}$ from major vessels (aorta, upper mediastinal vessels, and pulmonary artery extending to the tertiary bronchus), esophagus, heart, trachea, or pericardium $(15,16)$. Local failure was defined as growth following initial tumor shrinkage, or progression, on two consecutive follow-up scans (date of local failure 
was backdated to the earliest scan showing progression) or biopsy-proven recurrence. Nodal failure was defined as hilar, mediastinal, or supraclavicular nodal progression on follow-up imaging/exam or biopsy-proven. Distant failures were failures outside of the thorax, malignant pleural/ pericardial effusions, and disease in different lobes. Timeto nodal failure, distant failure or any-failure was defined as the time from the date of completion of SBRT to the date of nodal, distant, or any failure, respectively (with patients censored at death). Adding "survival" to the above timeto event functions adds death from any cause as an "event". Therefore, nodal failure-free survival, distant failure-free survival and any failure-free survival were defined as the time between SBRT completion and either; nodal, distant or any failure or death from any cause. Overall survival (OS) was defined as the time between SBRT completion and death from any cause. For all of these, patients were otherwise censored at the time of their last follow-up.

\section{Statistical considerations}

Cox regression was used to evaluate the time-to event outcomes and the select patient covariates of interest: tumor size, location, appearance, histology, stage, lymph node suspicion, radiation dose, and receipt of invasive mediastinal nodal staging. Both univariable and adequately powered multivariable models were evaluated. Whether or not a patient received invasive mediastinal nodal staging was of particular interest and this covariate was 'forced into' the multivariable models examined. When comparing covariates of interest by invasive mediastinal nodal staging status, the
Wilcoxon two-group test was used for continuous variables. For nominal categorical variables, Fisher's exact test was used. For ordinal categorical variables, the nonparametric Jonckheere-Terpstra method was used to test for ordered differences among categories. With this test, the null hypothesis is that the distribution of the response does not differ across the ordered categories. Kaplan-Meier plots were produced for time-to event functions by invasive mediastinal nodal staging status and the log-rank test was reported for the comparisons by invasive mediastinal nodal staging status. Two-sided $\mathrm{P}$ values less than 0.05 were considered statistically significant.

Statistical analyses were done using both SAS and R. SAS Version 9.4 is from the SAS Institute, Inc., Cary, NC, USA. $\mathrm{R}$ is an open source statistical programming language from the R Development Core Team (2019), R Foundation for Statistical Computing, Vienna, Austria.

\section{Ethical statement}

The study was conducted in accordance with the Declaration of Helsinki (as revised in 2013). The study was approved by Institutional Review Board of University of North Carolina Hospitals (IRB\# 14-2566) and individual consent for this retrospective analysis was waived.

\section{Results}

In this study, 158 patients were treated with SBRT at a total dose ranging from 32-60 Gy over 1-5 fractions (median dose of 48 Gy, median fractions of 4). Table 1

Table 1 Patient characteristics by invasive mediastinal nodal staging status

\begin{tabular}{|c|c|c|c|}
\hline & \multicolumn{2}{|c|}{ Invasive mediastinal nodal staging } & \multirow[b]{2}{*}{$P$ value } \\
\hline & No $(N=79)$ & Yes $(N=79)$ & \\
\hline Median age (range) & $71[46-87]$ & 77 [49-90] & 0.31 \\
\hline Gender & & & 0.50 \\
\hline Male & $39(49 \%)$ & $40(51 \%)$ & \\
\hline Female & 40 (51\%) & $39(49 \%)$ & \\
\hline Smoking history (pack years) & & & 0.25 \\
\hline Median (IQR) & 62 [43.5-80] & $50[40-75]$ & \\
\hline Prior lung cancer & & & 0.85 \\
\hline Yes & $20(25 \%)$ & $18(23 \%)$ & \\
\hline No & $59(75 \%)$ & $61(77 \%)$ & \\
\hline
\end{tabular}

Table 1 (continued) 
Table 1 (continued)

\begin{tabular}{|c|c|c|c|}
\hline & \multicolumn{2}{|c|}{ Invasive mediastinal nodal staging } & \multirow[b]{2}{*}{$P$ value } \\
\hline & No $(N=79)$ & Yes $(\mathrm{N}=79)$ & \\
\hline ECOG PS & & & 0.31 \\
\hline 0 & $34(43 \%)$ & $25(32 \%)$ & \\
\hline 1 & $35(45 \%)$ & $46(59 \%)$ & \\
\hline 2 & $9(12 \%)$ & $7(9 \%)$ & \\
\hline Tumor lobe (primary) & & & 0.29 \\
\hline RUL & $32(40 \%)$ & $21(27 \%)$ & \\
\hline RML & $2(2 \%)$ & $5(6 \%)$ & \\
\hline RLL & $14(18 \%)$ & $15(19 \%)$ & \\
\hline Tumor histology (primary) & & & 0.28 \\
\hline Adenocarcinoma & $28(36 \%)$ & $26(33 \%)$ & \\
\hline Squamous cell carcinoma & $27(34 \%)$ & $28(36 \%)$ & \\
\hline Other/non-diagnostic & $24(30 \%)$ & $25(31 \%)$ & \\
\hline Tumor location (primary) & & & $<0.0001$ \\
\hline Central & $10(13 \%)$ & $32(41 \%)$ & \\
\hline Peripheral & $69(87 \%)$ & $47(59 \%)$ & \\
\hline Clinical T-stage & & & 0.99 \\
\hline Solid & $69(87 \%)$ & $72(91 \%)$ & \\
\hline Ground glass opacity (GGO) & $7(9 \%)$ & $4(5 \%)$ & \\
\hline Mixed (part solid, part GGO) & $3(4 \%)$ & $3(4 \%)$ & \\
\hline PET/CT staging & & & 0.17 \\
\hline Yes & $72(91 \%)$ & 77 (97\%) & \\
\hline No & $7(9 \%)$ & $2(3 \%)$ & \\
\hline Suspicious LN on PET/CT & & & 0.23 \\
\hline Yes & $12(16 \%)$ & $19(25 \%)$ & \\
\hline No & $61(84 \%)$ & $58(75 \%)$ & \\
\hline Missing & 6 & 2 & \\
\hline Median SBRT dose (range) & $4,800[3,200-6,000]$ & $4,800[3,200-6,000]$ & 0.55 \\
\hline
\end{tabular}

IQR, interquartile range; ECOG PS, Eastern Cooperative Oncology Group Performance Status; T-Stage, AJCC Clinical Criteria; GGO, ground-glass opacity; "Suspicious LN on PET/CT" represents the presence of a suspicious lymph node on PET during workup. SBRT, stereotactic body radiation therapy. 
Table 2 Time-to event outcomes-3-year probabilities and median times with $95 \%$ confidence limits

\begin{tabular}{|c|c|c|c|c|}
\hline Time-to event outcome & Invasive mediastinal nodal staging & 3-year probability (95\% Cl) & Median in months $(95 \% \mathrm{Cl})$ & $P$ value \\
\hline Nodal failure-free survival & No & $0.55(0.43-0.66)$ & 40 [29-64] & \\
\hline \multirow[t]{2}{*}{ Nodal failure } & Yes & $0.84(0.72-0.91)$ & - & 0.1 \\
\hline & No & $0.69(0.56-0.79)$ & - & \\
\hline Any failure-free survival & No & $0.45(0.33-0.57)$ & 33 [19-53] & \\
\hline \multirow[t]{2}{*}{ Any failure } & Yes & $0.62(0.48-0.73)$ & $48\left(36,{ }^{*}\right)$ & 0.95 \\
\hline & No & $0.54(0.41-0.66)$ & $61(31, *)$ & \\
\hline Distant failure-free survival & Yes & $0.63(0.51-0.74)$ & $41[36,50]$ & 0.63 \\
\hline Distant failure & No & $0.83(0.71-0.73)$ & - & \\
\hline \multirow[t]{2}{*}{ Overall survival } & Yes & $0.65(0.52-0.75)$ & $43[36,62]$ & 0.65 \\
\hline & No & $0.67(0.54-0.76)$ & $63[39,70]$ & \\
\hline
\end{tabular}

*, empty cells not estimable; Nodal Failure: represents freedom from nodal failure; Any Failure: represents freedom from any failure; Distant Failure: represents freedom from distant failure.

shows patients' characteristics (including select tumor and treatment characteristics) stratified by invasive mediastinal nodal staging status. Prior to treatment, most patients 149/158 (or 94\%) underwent PET/CT staging and all patients underwent primary tumor biopsy with fiducial marker placement. Seventy-nine patients underwent biopsy using CT-guidance and 79 patients underwent navigational bronchoscopy for biopsy. All patients undergoing biopsy with bronchoscopy underwent EBUS-TBNA to sample mediastinal and hilar lymph nodes, while nodal assessment of patients undergoing CT-guided biopsy was limited to radiologic assessment with PET/CT. No patients underwent mediastinoscopy for staging. The two groups were comparable for most characteristics (see Table 1). However, patients in the CT-guided biopsy group were far more likely to have peripheral lesions than were the bronchoscopic biopsy group.

Median OS for all 158 patients was 50 months (95\% CI: 39-63); 79 patients died. Median follow-up for survivors was 33 months. OS was not significantly associated with invasive mediastinal nodal staging status $(\mathrm{P}=0.65)$. A crude comparison of the outcomes for the patients with and without invasive mediastinal nodal staging is shown in Table 2, and there are no evident differences. Comparisons of Kaplan-Meier plots for these same endpoints are shown graphically in Figures 1-7.

Univariable Cox regression models were used to evaluate possible associations of patient characteristics of interest with OS. Only the number of pack years smoking $(\mathrm{P}=0.001)$, presence of suspicious lymph nodes on PET/CT $(\mathrm{P}=0.05)$, and radiation dose $(\mathrm{P}=0.05)$ were significantly associated with OS. Pack years smoking was also significant in nodal failure-free survival $(\mathrm{P}=0.003)$, distant failure-free survival $(\mathrm{P}=0.001)$, and any failure-free survival $(\mathrm{P}=0.001)$. Radiation dose was significant in any failure-free survival and distant failure-free survival $(\mathrm{P}=0.002$ and $\mathrm{P}=0.04$ respectively). The presence of suspicious lymph nodes on PET/CT was significant with nodal failure-free survival $(\mathrm{P}=0.02)$. In fact, those who had suspicious lymph nodes were 3.1 times more likely to have nodal failure $(\mathrm{OR}=3.1,95 \% \mathrm{CI}: 1.3-7.5$, $\mathrm{P}=0.02)$.

In multivariable Cox regression models where sampling modality (CT-guidance without invasive mediastinal nodal staging or bronchoscopy with) was 'forced' into the model, the fit of the model was worse, meaning that the single variable model fit best for each variable of interest and each time-to event outcome. Knowing the presence or absence of invasive mediastinal nodal staging did not add information 


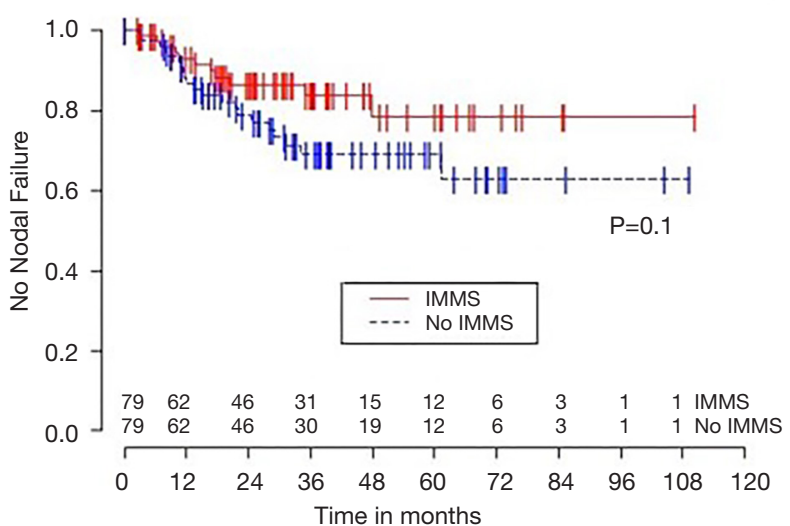

Figure 1 Freedom from nodal failure. Figure shows the rate of not having a nodal failure in the study population.

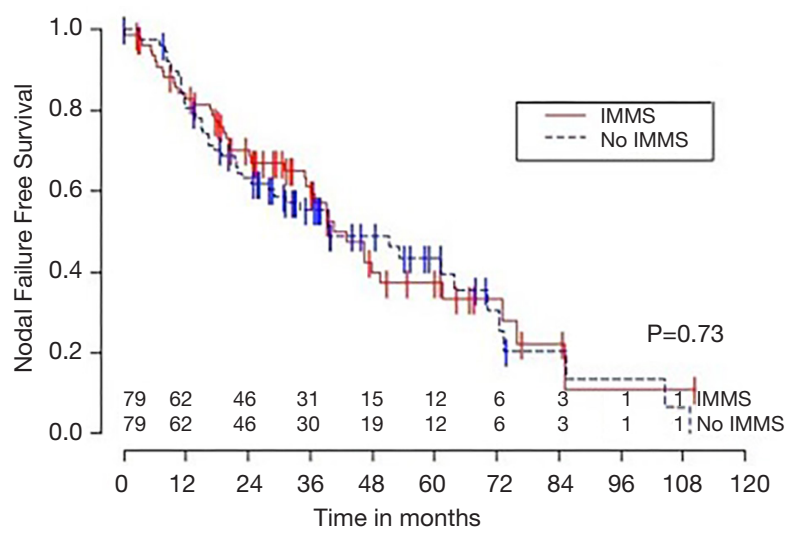

Figure 2 Nodal failure-free survival. Figure shows the nodal failure-free survival rate in the study population.

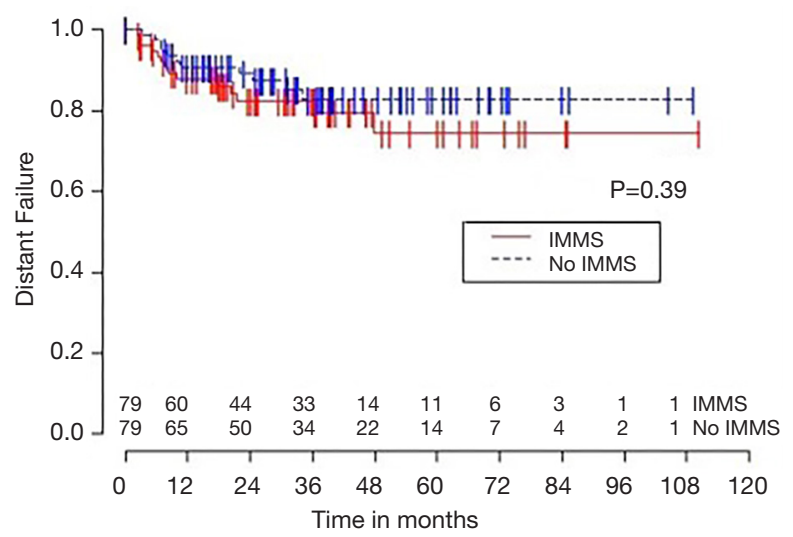

Figure 3 Freedom from distant failure. Figure shows the rate of not having a distant failure in the study population.

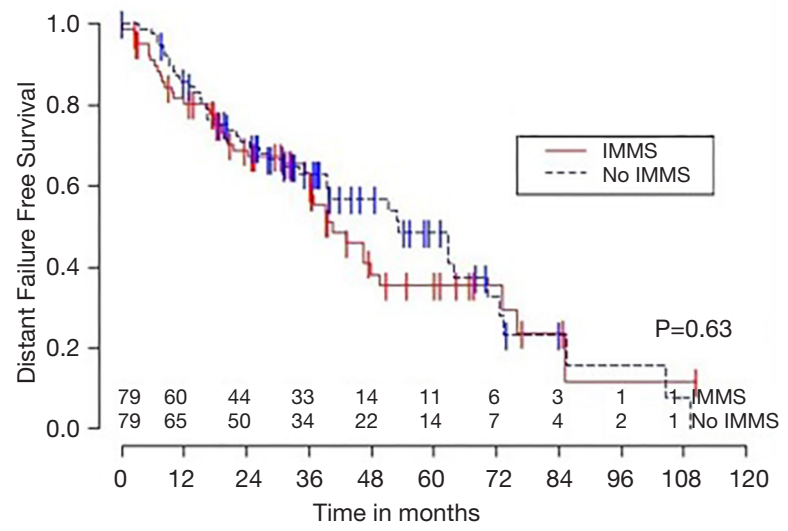

Figure 4 Distant failure-free survival. Figure shows the distant failure-free survival rate in the study population.

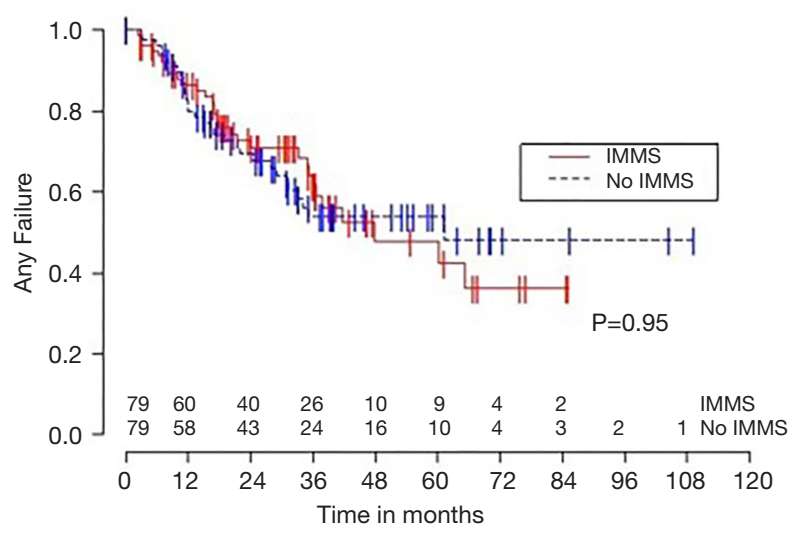

Figure 5 Freedom from any failure. Figure shows the rate of not having disease failure in the study population.

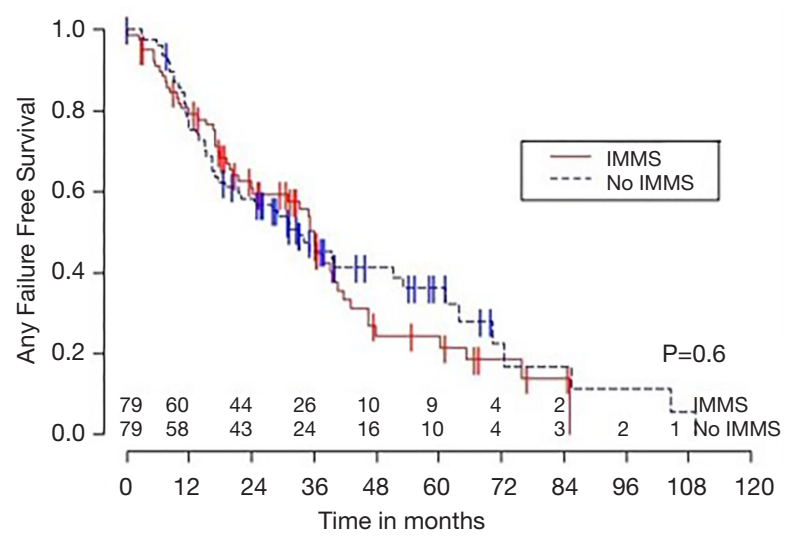

Figure 6 Any failure-free survival. Figure shows the failure-free survival rate in the study population. 


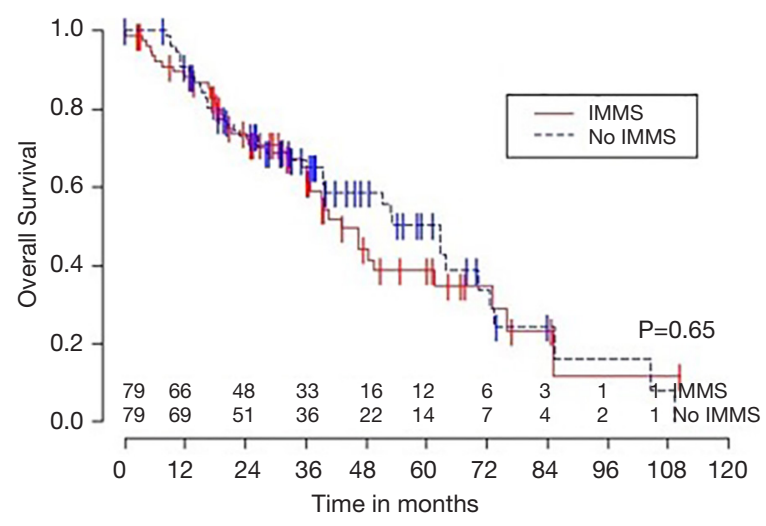

Figure 7 Overall survival. Figure shows the overall survival rate in the study population.

when combined with any of the other covariates of interest concerning any of the time-to event outcomes.

\section{Discussion}

The majority of medically inoperable early-stage NSCLC patients undergo non-invasive nodal staging with PET/ CT prior to SBRT with or without the addition of invasive mediastinal nodal staging. While PET/CT has proven to be an accurate method of nodal assessment with negative predictive value $>80-90 \%(5,10)$, it is important to fully understand whether outcomes are impacted when selected in lieu of invasive sampling techniques. The clinical benefit of invasive nodal staging in this patient population has not been well-studied. To fulfill this knowledge gap, we analyzed data from a large, diverse, cohort of patients resulting in several notable findings that add to the existing literature.

First, our study suggests that in the PET/CT era, medically inoperable patients who undergo SBRT for early-stage NSCLC, have similar outcomes regardless of receipt of invasive mediastinal nodal staging. There were no significant differences in nodal failure rates, failurefree survival or OS between those undergoing invasive mediastinal nodal staging (EBUS-TBNA at time of navigational bronchoscopy) and those undergoing CTguided biopsy without subsequent invasive mediastinal nodal staging. These findings are also consistent with the available literature as Schonewolf et al. similarly found no differences in survival or recurrence patterns in their patients receiving invasive mediastinal nodal staging compared to PET/CT alone in a retrospective analysis of patients on prospectively collected SBRT registries at two academic institutions (17). Given the uncertain benefit of invasive mediastinal nodal staging, it certainly seems reasonable to omit invasive mediastinal nodal staging in select patients with negative nodes by PET/CT, especially in those who have comorbidities that might increase their risk of morbidity with invasive nodal staging procedures.

Nevertheless, invasive mediastinal nodal staging may still be helpful in some subgroups of patients. For example, patients with a particularly good prognosis (e.g., medically operable with fewer comorbidities) could theoretically benefit from the more accurate staging given their potential for extended longevity. Additionally, a variety of tumorrelated factors may indicate a higher risk of microscopic nodal involvement (e.g., >10\%) including central tumor location and T2 tumor size with solid appearance on CT (8,18-22), which might warrant invasive mediastinal nodal staging. We did not find a significant association with increased nodal failure in these patients; however, such patients were a small minority in our study. Given that the majority of our patients had T1 (125/158 or 79\%) and peripheral (116/158 or $73 \%)$ tumors, our findings might not be applicable to larger and more central tumors, as these patients have increased risk of nodal involvement and may benefit more from the accuracy of invasive mediastinal nodal staging.

Second, the presence of suspicious lymph nodes on PET/CT was associated with worse outcomes (e.g., regarding nodal failure, nodal failure-free survival, and OS). A similar finding has been shown in the setting of surgery for early-stage pathologically node-negative lung cancer, as the pre-operative PET/CT findings in the nodes were predictive for post-operative outcomes (23). The presence of suspicious lymph nodes on PET/CT should generally be an indication for invasive mediastinal nodal staging, even to be considered if the pattern seems more "reactive" or consistent with granulomatous disease (e.g., symmetric uptake in the bilateral hilum and mediastinum). Since not all patients are optimal candidates for invasive procedures, PET/CT findings can help provide guidance in situations in which the risks of invasive sampling may otherwise come close to, or slightly outweigh, the initial perceived benefit.

Third, the marked differences between the failure and failure-free survival outcomes and similarities between failure-free survival and OS outcomes (see Figures 1-6), emphasizes the relatively high rate of intercurrent deaths in this population of patients with significant comorbidities. The event of death appears to be the driver in these time- 
to event survival comparisons. Such competing risks are common in studies of patients with NSCLC undergoing SBRT.

Strengths of our study include the large sample of patients with a wealth of available data on patient, tumor, and treatment characteristics. Additionally, our institution is a large tertiary center with patients coming from across North Carolina and the surrounding states. Therefore, it appears that our sample should be representative of patients with this disease.

There are several limitations of our study. First, patients were not randomized based on receipt of invasive mediastinal nodal staging, and thus the comparisons might be biased. To address this, we carefully compared the known prognostic factors in the two groups and they appear to be reasonably well-balanced (Table 1). However, the patients receiving invasive mediastinal nodal staging did more often have central tumors and suspicious lymph nodes on PET/CT. The MVA was used as a means to adjust for the impact of these imbalances. This is a common approach in these types of clinical studies. Second, data was extracted retrospectively from medical records. Nevertheless, we were able to capture the desired data, and fewer than $1 \%$ of the data elements were missing from the analysis. Third, the categorization of suspicious lymph nodes was done qualitatively, as our institution does not report standardized uptake values for PET/CT. Instead, the uptake is compared to blood pool and hepatic parenchyma, leading to nodal characterization of "suspicious for reactive vs. metastasis". However, this is a commonly used and well-accepted approach to interpreting imaging tests. Fourth, this study population consists only of patients treated with SBRT. By definition, patients with pathologic nodal involvement during invasive mediastinal nodal staging are not included (as these patients would not be candidates for SBRT). In this regard, the patients undergoing invasive mediastinal nodal staging might be considered to be "more favorable" than the patients not undergoing invasive nodal staging. The failure to observe better outcomes in the group undergoing invasive mediastinal nodal staging may reflect their higher rate of other unfavorable clinical factors (e.g., tumor location, CT/PET findings), the impact of intercurrent diseases and comorbidities, and/or an effect of modest sample size. Fifth, new lung lesions in the current study were classified as metachronous primary tumors and not distant metastases [as has been done by some others, e.g., Schonewolf (17)]. While this would affect the reported distant failure-free survival, this would not affect nodal failure-free survival. We specifically elected to look at a variety of outcome measures in order to consider this issue.

In conclusion, our study showed roughly equivalent outcomes between early-stage NSCLC patients receiving invasive $v s$. non-invasive mediastinal nodal staging with PET/CT prior to undergoing SBRT. These results might help inform decision-making on invasive nodal staging, particularly in patients who might be marginal candidates for invasive procedures. It is important to note however, that this study focused on medically inoperable patients with mostly small peripheral tumors and might not be generalizable to medically operable patients (e.g., with fewer comorbidities that would impact tolerance of invasive procedures or limit survival from non-oncologic conditions) or to patients with large or central primary tumors. Future work will focus on the radiographic characterization of the lesions and re-evaluation of pre-treatment PET/CT to guide future decision-making for invasive mediastinal nodal staging prior to SBRT.

\section{Acknowledgments}

Funding: Support in part by funds from the Lineberger Comprehensive Cancer Center.

\section{Footnote}

Reporting Checklist: The authors have completed the STROBE reporting checklist. Available at http://dx.doi. org/10.21037/jtd-20-2808

Data Sharing Statement: Available at http://dx.doi. org/10.21037/jtd-20-2808

Conflicts of Interest: All authors have completed the ICMJE uniform disclosure form (available at http://dx.doi. org/10.21037/jtd-20-2808). The authors have no conflicts of interest to declare.

Ethical Statement: The authors are accountable for all aspects of the work in ensuring that questions related to the accuracy or integrity of any part of the work are appropriately investigated and resolved. The study was conducted in accordance with the Declaration of Helsinki (as revised in 2013). The study was approved by Institutional Review Board of University of North Carolina Hospitals (IRB\# 14-2566) and individual consent for this retrospective analysis was waived. 
Open Access Statement: This is an Open Access article distributed in accordance with the Creative Commons Attribution-NonCommercial-NoDerivs 4.0 International License (CC BY-NC-ND 4.0), which permits the noncommercial replication and distribution of the article with the strict proviso that no changes or edits are made and the original work is properly cited (including links to both the formal publication through the relevant DOI and the license). See: https://creativecommons.org/licenses/by-nc-nd/4.0/.

\section{References}

1. Chang JY, Senan S, Paul MA, et al. Stereotactic ablative radiotherapy versus lobectomy for operable stage I nonsmall-cell lung cancer: a pooled analysis of two randomised trials. Lancet Oncol 2015;16:630-7.

2. Zheng X, Schipper M, Kidwell K, et al. Survival outcome after stereotactic body radiation therapy and surgery for stage I non-small cell lung cancer: a meta-analysis. Int J Radiat Oncol Biol Phys 2014;90:603-11.

3. Yu XJ, Dai WR, Xu Y, et al. Survival Outcome after Stereotactic Body Radiation Therapy and Surgery for Early Stage Non-Small Cell Lung Cancer: A MetaAnalysis. J Invest Surg 2017. [Epub ahead of print]. doi: 10.1080/08941939.2017.1341573.

4. Senthi S, Lagerwaard FJ, Haasbeek CJ, et al. Patterns of disease recurrence after stereotactic ablative radiotherapy for early stage non-small-cell lung cancer: a retrospective analysis. Lancet Oncol 2012;13:802-9.

5. Silvestri GA, Gonzalez AV, Jantz MA, et al. Methods for staging non-small cell lung cancer: Diagnosis and management of lung cancer, 3rd ed: American College of Chest Physicians evidence-based clinical practice guidelines. Chest 2013;143:e211S-50S.

6. Fernández-Villar A, Botana M, Leiro V, et al. Validity and reliability of transbronchial needle aspiration for diagnosing mediastinal adenopathies. BMC Pulm Med 2010;10:24.

7. Rakha EA, Naik V, Chaudry Z, et al. Cytological assessment of conventional transbronchial fine needle aspiration of lymph nodes. Cytopathology 2010;21:27-34.

8. Gao SJ, Kim AW, Puchalski JT, et al. Indications for invasive mediastinal staging in patients with early nonsmall cell lung cancer staged with PET-CT. Lung Cancer. 2017;109:36-41.

9. Kim YK, Lee KS, Kim BT, et al. Mediastinal nodal staging of nonsmall cell lung cancer using integrated 18F-FDG PET/CT in a tuberculosis-endemic country: diagnostic efficacy in 674 patients. Cancer 2007;109:1068-77.

10. Li X, Zhang H, Xiang L, et al. Mediastinal lymph nodes staging by 18F-FDG PET/CT for early stage non-small cell lung cancer: a multicenter study. Radiother Oncol 2012;102:246-50.

11. Farjah F, Flum DR, Ramsey SD, et al. Multi-modality mediastinal staging for lung cancer among medicare beneficiaries. J Thorac Oncol 2009;4:355-63.

12. Ost DE, Niu J, Elting LS, et al. Quality gaps and comparative effectiveness in lung cancer staging and diagnosis. Chest 2014;145:331-45.

13. Faris N, Yu X, Sareen S, et al. Preoperative Evaluation of Lung Cancer in a Community Health Care Setting. Ann Thorac Surg 2015;100:394-400.

14. Goldstraw P, Chansky K, Crowley J, et al. The IASLC Lung Cancer Staging Project: Proposals for Revision of the TNM Stage Groupings in the Forthcoming (Eighth) Edition of the TNM Classification for Lung Cancer. J Thorac Oncol 2016;11:39-51.

15. Timmerman R, McGarry R, Yiannoutsos C, et al. Excessive toxicity when treating central tumors in a phase II study of stereotactic body radiation therapy for medically inoperable early-stage lung cancer. J Clin Oncol 2006;24:4833-9.

16. Korzets Ceder Y, Fenig E, Popvtzer A, et al. Stereotactic body radiotherapy for central lung tumors, yes we can! Radiat Oncol 2018;13:77.

17. Schonewolf CA, Verma V, Post CM, et al. Outcomes of invasive mediastinal nodal staging versus positron emission tomography staging alone for early-stage non-small cell lung cancer treated with stereotactic body radiation therapy. Lung Cancer 2018;117:53-9.

18. Lee PC, Port JL, Korst RJ, et al. Risk factors for occult mediastinal metastases in clinical stage I non-small cell lung cancer. Ann Thorac Surg 2007;84:177-81.

19. Tsutani $Y$, Miyata $Y$, Yamanaka T, et al. Solid tumors versus mixed tumors with a ground-glass opacity component in patients with clinical stage IA lung adenocarcinoma: prognostic comparison using high-resolution computed tomography findings. J Thorac Cardiovasc Surg 2013;146:17-23.

20. Koike T, Koike T, Yamato Y, et al. Predictive risk factors for mediastinal lymph node metastasis in clinical stage IA non-small-cell lung cancer patients. J Thorac Oncol 2012;7:1246-51.

21. Ketchedjian A, Daly BD, Fernando HC, et al. Location as an important predictor of lymph node involvement for pulmonary adenocarcinoma. J Thorac Cardiovasc Surg. 
2006;132:544-8.

22. Al-Sarraf N, Aziz R, Gately K, et al. Pattern and predictors of occult mediastinal lymph node involvement in non-small cell lung cancer patients with negative mediastinal uptake on positron emission tomography. Eur
J Cardiothorac Surg 2008;33:104-9.

23. Xie L, Saynak M, Veeramachaneni NK, et al. Non-small cell lung cancer: prognostic importance of positive FDG PET findings in the mediastinum for patients with $\mathrm{N} 0-\mathrm{N} 1$ disease at pathologic analysis. Radiology 2011;261:226-34.

Cite this article as: Mullins BT, Moore DT, Rivera MP, Marks LB, Akulian J, Pearlstein KA, Wang K, Burks AC, Weiner AA. The impact of pathologic staging of the hilar/mediastinal nodes on outcomes in patients with early-stage NSCLC receiving stereotactic body radiotherapy. J Thorac Dis 2021;13(2):1045-1054. doi: $10.21037 /$ jtd-20-2808 